\title{
Clustering Initialization Based on Spatial Information for Speaker Diarization of Meetings
}

\author{
J. Luque, C. Segura, J. Hernando \\ Technical University of Catalonia (UPC), TALP Research Center, Barcelona (Spain) \\ luqueet.sc.upc.edu
}

\begin{abstract}
This paper proposes an initialization for an agglomerative system applied to speaker diarization in the meeting environment. The initialization is based on a previous clustering of the temporal sequence generated by the estimation of the Time Delay of Arrival (TDOA) among pair of sensors. That initial clustering has the purpose of obtaining initial classes with speaker information from a sole speaker. The aim is to ensure the purity of the initial segments based on the position of the speakers in a meeting along time. The TDOA initialization was tested with the dataset used in the RT07s evaluation where an improvement of the diariazation error rate is obtained with respect to the classical uniform initialization. The most of the experiments show that the purity of the beginning segments leads to a better clustering on the posterior hierarchical strategy based on cepstral features.
\end{abstract}

Index Terms: Speaker diarization, speaker segmentation, speaker clustering, cluster initialization

\section{Introduction}

Speaker diarization consists in segmenting and labelling an unknown set of speakers in a continuous audio stream. Diarization is a pattern recognition problem, since it strives to find the most likely categorization of a sequence of acoustic observations. Such information becomes useful in many applications like as speaker indexing, information retrieval and as a pre-processing for the speech content transcription, since regions of different nature can be handled in different way [1]. In a real situation such as meetings the problem becomes a challenge so the system has to face a lot of troubles such a variable environment, reverberations or overlapped speech. For instance, in the NIST Rich Transcription Evaluations [2] there is no prior knowledge available on the room setup, about how many speakers are there or their characteristics.

Speaker diarization includes a segmentation step with the purpose of finding the speaker change points and a clustering step carrying out the labelling. Overall, it discriminates between different speakers, creating homogeneous segments along time and joining them in clusters from the same speaker. Two main approaches can be found in the bibliography which face this problem. The first one handles the segmentation and the clustering in separated steps, and the other one deals with them in an integrated way. In the step-by-step approach, called sequential systems, a distance measure between two adjacent points of the acoustic observations is applied along the time. The Generalized Likelihood Ratio (GLR) and the Bayesian Information

This work has been funded by the the Spanish project SAPIRE (TEC2007-65470)
Criterion (BIC) are the most popular techniques employed to estimate the speaker change detection [3]. In the integrated approach, Hidden Markov Models (HMM) [4] perform the segmentation and the clustering steps at the same time in an iterative top-down strategy. The so called agglomerative clustering is the most popular technique in speaker diarization and it is also used in this paper.

The initialization in the agglomerative clustering has a great importance despite of the multiple iterations of segmentation and merging of the data. In the uniform initialization no constraint is applied to the prior clusters. Acoustic models are trained from such data and the iterative segmentation assigns the observations in homogeneous clusters. However, in some cases, acoustic segments from more than one speaker remain in their originally-assigned cluster throughout the clustering process or clusters containing information from various speakers are erroneously merged. In such situations we end up with an increase in the diarization error rate (DER) which is difficult to reduce during the clustering process. The use of a standard k-means algorithm [4] to find the cluster initialization leads to even worse results than with the linear initialization algorithm. Other initialization based on the purity of initial cluster and BIC segmentation was suggested in [9].

In this paper a TDOA-based initialization for a hierarchical diarization system is introduced. In a multi-microphone environment, the use of redundant signals can improve the classical diarization systems. On the one hand, this information can be used for signal enhancement by applying a delay\&sum algorithm as in [6]. On the other hand, the speaker localization can directly perform the speaker segmentation and the clustering as in [7] where a diarization based on Direction Of Arrival (DOA) information is proposed. In other paper [8], the diarization is performed in an agglomerative way mixing the cepstral and the delay observations. It outperforms the experiments with only spectral features.

The novel TDOA clustering proposed is based on the analysis of the temporal statistics of the distance among TDOA pairs. The aim is to obtain speakers clusters from a unique person. That cluster purity is based on the hypothesis that if a speech is produced from the same location during a certain period of time, it might come from the same speaker. The resulting TDOA clustering becomes the initial clustering condition for the integrated speaker diarization based on a HMM modeling of cepstral features. This novel initialization is compared with the classical uniform initialization. In addition, it is also studied in both a mono-channel and a multi-channel diarization systems. The latter performs the speaker diarization over an enhanced signal obtained by means a weighted-delay\&sum algorithm. 


\section{TDOA-based clustering}

In order to estimate the TDOA between segments corresponding to two microphones, we used a modified version of the Generalized Cross Correlation with phase transform GCCPHAT [10]. GCCPHAT has been widely used in the acoustic localization task [11] and in the blind signal separation field [12]. Moreover, it is known to perform robustly in reverberant environments.

TDOA features by themselves permit short-term speaker segmentation by analyzing the delays steadiness. It can be employed for the tracking and the segmentation of individuals in a $2 \mathrm{D}$ or $3 \mathrm{D}$ metric space by making use of the geometrical information between microphones. Unfortunately, for the NIST task where microphone locations are not available, a different strategy is needed.

Anyway, the delays between microphone pairs are related to a positions in the 3D space by a non-linear hyperbolic function. Without the knowledge of the microphone geometry, we can only conclude that a displacement of an active speaker in the room yields to different shifts in the estimated delays of each microphone pair. Such relationship with the real 3D geometry depends on the distance between microphones and the relative position of the speaker. Thus, the total dynamic range of the TDOAs of every microphone pair is associated to the maneuvering of the speakers in the room. With this assumption and analyzing the distribution of the TDOA values along time, a set of possible locations can give us the information of the speech events during a recording. The continuity of such values within a segment of time might be associated to the same speaker position. When a speaker change occurs, the TDOA changes and its value is associated to the new speaker location. Moreover, if a set of TDOA is available a smoothing of this strategy can be performed rejecting the wrong estimations of the time delays.

In terms of the number of microphones $K$ in the recording, the total number of possible TDOAs at each time $t$ is given by the combinatorial number $\left(\begin{array}{c}K \\ 2\end{array}\right)=N$. We define the TDOA space as the set of points $\vec{\tau}_{t}=\left(\tau_{1 t}, \tau_{2 t}, \ldots, \tau_{N t}\right)$ where each $\tau_{i t}$ is the estimated TDOA from a given pair of microphones $i$ in time $t$. The range of values that can reach each component of $\vec{\tau}_{t}$ differs since the real distance between microphone pairs might be different. To avoid this problem each component of $\vec{\tau}_{t}$ is normalized, independently for each component, based on the dynamic range of the TDOA values during an interval of time $T$ as follows:

$$
\tau_{\text {itnorm }}=\frac{\tau_{i t}}{\left(\max _{t \in T}\left(\tau_{i t}\right)-\min _{t \in T}\left(\tau_{i t}\right)\right)}, \quad i=1, \ldots, N
$$

After this normalization, the distance between two points $d\left(\vec{\tau}_{t}, \vec{\tau}_{t+1}\right)$ is defined by means the Euclidean distance. Next the tracking and segmentation of moving speakers can be obtained by applying classical spatial data association and clustering techniques [13].

However, the Euclidean distance between $\vec{\tau}_{t}$ vectors is highly sensitive due the TDOA estimation errors. Thus, we assume that if a percentage of the TDOAs are not changing during a time segment, the same person is speaking. With such assumption we propose to compute the distance in the normalized TDOA space as the Euclidean distance in a subset of $N$ with dimension $n<N$. That subset represents the TDOA components of $\vec{\tau}$ which have the lowest variation in each time step. In order to select the $n$ best TDOAs, the difference in two time steps, $\overrightarrow{\tau_{\mathbf{t}}}-\vec{\tau}_{t+1}$, is computed. Next, those $n$ closest TDOAs are

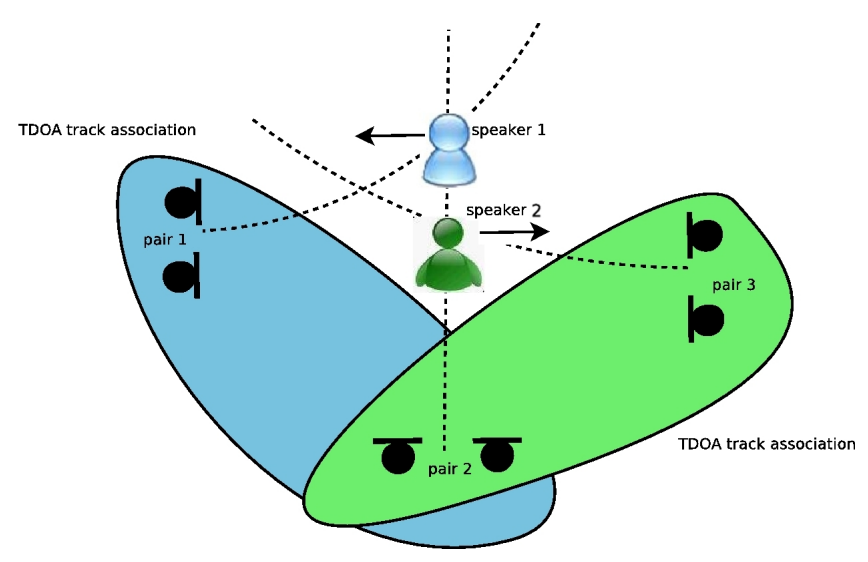

Figure 1: Two speaker example where two tracks are associated to the same observated TDOAs, involving 3 microphone pairs. The arrow indicates the speech source orientation and, for this example, the distance is defined with dimension $n=2$

selected based on a threshold and they are employed to compute the Euclidean distance as,

$$
d\left(\vec{\tau}_{t}, \vec{\tau}_{t+1}\right)=\frac{1}{n} \sum_{i=1}^{n}\left(\tau_{i t}-\tau_{i(t+1)}\right)^{2}
$$

Such TDOAs are more likely to focus on a particular source. Note that this criterion is evaluated in each $t$ so the $n$ selected components of $\vec{\tau}$ could be different at each iteration.

Finally, with the purpose of capturing the temporal evolution of distances $d\left(\vec{\tau}_{t}, \vec{\tau}_{t+1}\right)$ and to associate several speakers detections along time, we track the temporal variations of $\vec{\tau}_{t}$ in the normalized TDOA subset in the following way:

- Assignation of new TDOAs to their corresponding active tracks, if they exist. The incoming TDOA estimates are associated to tracks based on the distance.

- Check the distance between tracks. Similar tracks are merged into one model. Tracks without an input association during a period of time are marked as non-active.

- Search of new tracks from TDOA history based on a distance threshold. Coincidence with non-active tracks are taken into account. The potential tracks are tested with deactivated tracks based on both TDOA distance and an exponential function of the elapsed time since the deactivation.

With this strategy, the non-active tracks are more likely to be merged with the new potential track. That assumption is based on the hypothesis that the tracking of a person that is not speaking may lead to errors since the person might have moved to other position losing the association between the speaker and his observable TDOAs. A time constrain is also imposed on the minimum duration of the tracking, fixing it to 3 seconds. Tracks with less duration are not considered.

As a example, the Figure 1 shows an hypothetical case involving two persons that are speaking simultaneously. We assume that due to the orientation of the speakers, the estimated TDOA at the microphone pair 1 is steered to person 1 and the TDOA at the pair 3 focus to speaker 2 , while the pair of microphones 3 has a TDOA that points to both speakers. In this hypothetical case and using $n=2$, two overlapped tracks are associated to the speakers and the set of TDOA pairs. So the algorithm also detects overlapped speakers in the recording. 


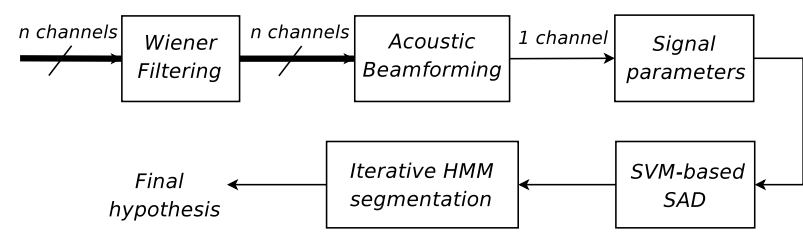

Figure 2: Brief scheme of the UPC implementation of the RT07s diarization system

\section{Cepstrum-based diarization}

The Figure 2 depicts an overall scheme of the diarization system submitted to previous RT07s [2] evaluation. Two different systems are defined in this work, the single distant microphone (SDM) approach and the multiple distant microphone (MDM) approach. The first one performs the diarization on a monochannel audio stream whereas the second one makes use of the TDOA, not only for cluster initialization but also for speech enhancement. For the SDM case, the reference channel used for diarization is given by NIST. For the MDM approach an enhanced signal is obtained by means of a weighted-delay\&sum of a reference channel in the same manner as in [6]. First of all the input signal is first Wiener filtered. Next, in both systems, the data is parameterized using $19 \mathrm{Mel}$ Frequency Cepstral Coefficients (MFCC). Such features are analyzed by a Speech Activity Detector (SAD) in order to obtain the speech segments which will be fed into the agglomerative algorithm. The nonspeech segments will be ignored for further processing. Finally, an iterative top-down clustering algorithm based on an HMM and a BIC metric distance among clusters is applied to the data. It joints the segments which belongs to the same speaker and finds out the number of speakers in the recording by means of a BIC-based stopping criterion.

In the case of the uniform initialization, the system starts with a homogeneous splitting of the whole data among an initial number of clusters equal to the initial number of states. This automatic selection of the number of clusters $\left(K_{\text {init }}\right)$ is defined as,

$$
K_{\text {init }}=\frac{N}{G_{\text {init }} C C R}
$$

by taking into account the total amount of data available per cluster $(N)$, the number of Gaussian mixtures initially assigned to each cluster $\left(G_{\text {init }}\right)$ and the Cluster Complexity Ratio $(C C R)$. The $C C R$ is a constant value fixed across all meetings that defines the number of frames per Gaussian.

In the case of the TDOA initialization, the initial number of clusters is obtained by the clustering of the speaker positions. The TDOA clustering system automatically selects those TDOA clusters which show a low distance in the normalized TDOA space and satisfy the condition of high stability within a temporal window. Unlike the uniform initialization, this selective clustering does not define a partition of the whole recording since not all the speech time is assigned to the clusters. In addition, a constrain to the total amount of clusters is imposed to avoid overclustering and to reduce the computational cost of the iterative approach. Therefore, the initialization is restricted to a minimum and a maximum of 30 and 40 clusters respectively. Moreover, before to apply the initial TDOA clusters, the segments defined by overlapped clusters are not considered in the TDOA initialization.

Once the intitial clustering is performed, each cluster is modelled by a Mixture of Gaussians, fitting the probability distribution of the features which belong to each cluster by the classical Expectation-Maximization (EM) algorithm. The $C C R$ constant is fixed to 7 seconds of data per Gaussian and the initial number of Gaussians per model $\left(G_{\text {init }}\right)$ is set to 5 . After an initial decoding of the observation sequence through a Viterbi algorithm a new clustering is obtained. A modified BIC-based metric [4] is used to decide the most likely pair of clusters to merge. The obtained segmentation defines a new number of clusters/states which will be retrained. In this work, a threshold depending on the standard deviation of the BIC value between pair of cluster is applied to decide which clusters to merge. Thus, the system could merge more than one pair of classes per each iteration in the case the BIC value for the pair excess the threshold. At each iteration $j$, the number $M_{i}^{j}$ of Gaussian mixtures to model cluster $i$ is updated by

$$
M_{i}^{j}=\operatorname{round}\left(\frac{N_{i}^{j}}{C C R}\right)
$$

where $N_{i}^{j}$ is the number of frames belonging to the cluster $i$. When two segments are merged, a new segment model is also trained joining all the features from the merged segments and taking into account the $C C R$ value to select the model complexity. This procedure is iterated until the stopping criterion is reached. Finally, at the last iteration each remaining state represents a different speaker. A detailed description of the system can be found in [14].

\section{Experiments}

In order to assess the proposed TDOA initialization algorithm, it is compared to the uniform initialization in the framework of the agglomerative speaker diarization system described in section 3. Both initialization techniques are compared using the data distributed for the NIST Rich Transcription 2007 Spring meeting Recognition Evaluation, RT07s [2]. It consists of excerpts from multi-party meetings in English collected at eight different sites at various time periods. From each meeting only an portion of 20 minutes is evaluated. The number of microphones available for each recording ranges from 3 to 16. The diarization system is applied to the single reference channel given by NIST, SDM condition, or to the enhanced signal from all available microphones, MDM condition.

To measure the performance of the proposed approaches, the NIST evaluation software [2] for speaker diarization is used. This metric, called Diarization Error Rate (DER) obtains the error as the percentage of time where the system labels are wrong looking at the overall accuracy. Additionally, the speaker error time (SER), which is responsable for the speaker identification error time is reported. DER includes the SER error but it takes also into account the missed speaker time error and the false alarm speaker time error.

Tables 1 reports the results obtained by the TDOA initialization in both conditions on the RT07s data. Overall, the use of the TDOA initialization reduces the global DER as well as the SER. The lowest DER $15.70 \%$ is obtained by the MDM system with the TDOA initialization (MDM-TDOA) improving a $4 \%$ relative with respect the uniform initialization. In the SDM system, the TDOA initialization also performs better with a $10 \%$ of relative improvement. However, the partial results per recording show a high variability of the DER and some results show an improvement in the SDM system but not in the MDM, and viceversa. The reduction of the SER also shows the benefit of the initial TDOA clustering. The initial estimations of 
Table 1: Diarization performance

\begin{tabular}{|c|c|c|c|c|c|c|c|c|}
\hline & \multicolumn{4}{|c|}{ SDM } & \multicolumn{4}{|c|}{ MDM } \\
\hline NAME SHOW & \multicolumn{2}{|c|}{$\%$ SER } & \multicolumn{2}{|c|}{$\% \mathrm{DER}$} & \multicolumn{2}{|c|}{$\%$ SER } & \multicolumn{2}{|c|}{$\%$ DER } \\
\hline & TDOA & UNI & TDOA & UNI & TDOA & UNI & TDOA & UNI \\
\hline CMU_20061115-1030 & 5.5 & 24.9 & 17.20 & 38.26 & 7.3 & 9.4 & 18.92 & 21.04 \\
\hline CMU_20061115-1530 & 1.3 & 13.7 & 8.37 & 21.27 & 6.3 & 9.5 & 13.42 & 16.61 \\
\hline EDI_20061113-1500 & 11.9 & 24.6 & 23.29 & 46.63 & 19.5 & 29.8 & 30.85 & 41.15 \\
\hline EDI_20061114-1500 & 13.6 & 12.2 & 19.83 & 29.02 & 9.9 & 11.0 & 16.10 & 17.26 \\
\hline NIST_20051104-1515 & 2.0 & 3.9 & 7.45 & 8.31 & 5.7 & 1.2 & 11.12 & 6.63 \\
\hline NIST_20060216-1347 & 29.8 & 2.5 & 34.28 & 6.90 & 7.1 & 1.8 & 11.56 & 6.33 \\
\hline VT_20050408-1500 & 12.4 & 6.6 & 18.21 & 12.36 & 1.1 & 7.1 & 6.92 & 12.92 \\
\hline VT_20050425-1000 & 21.1 & 7.1 & 28.97 & 15.44 & 10.5 & 3.7 & 18.39 & 11.64 \\
\hline ALL & 12.3 & 11.8 & 19.73 & 21.99 & 8.2 & 9.0 & 15.70 & 16.50 \\
\hline
\end{tabular}

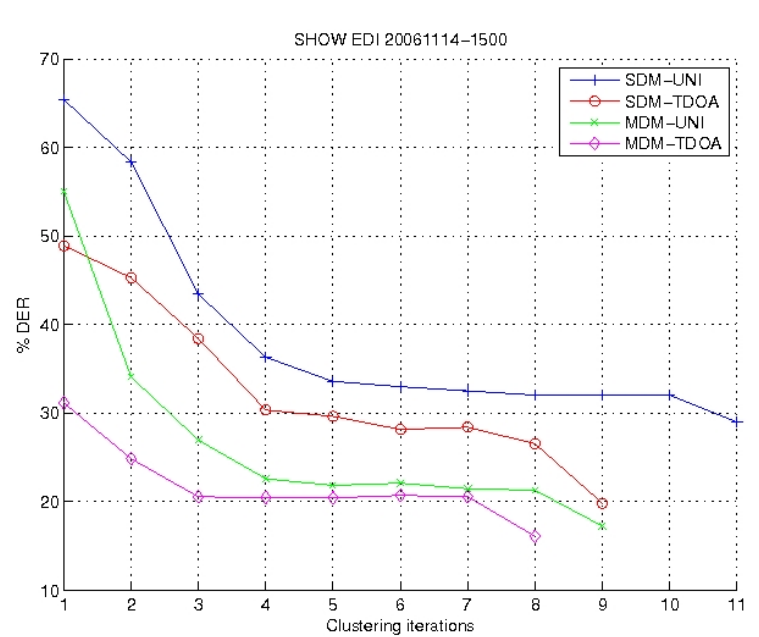

Figure 3: DER evolution per iteration of the recording EDI_20061114-1500. It shows the improvement applying the TDOA initialization in both SDM and MDM systems

the speaker models are more accurate which results in a SER reduction in the most of the recordings.

In Figure 3 a recording which shown a good performance in the most of the situations was selected to report its DER evolution. The error obtained in the first iteration is directly related with the way of initialization. The lowest DER is obtained in the TDOA initialization case. This improvement remains constant in the next iterations which illustrates the importance of the initialization way of the agglomerative clustering.

\section{Conclusions}

In this paper we have proposed a novel algorithm for the initialization of an agglomerative clustering system in the task of speaker diarization of meetings. The presented algorithm analyzes the TDOA distribution during a recording and exploits the most likely and stable pairs of TDOA to obtain an initial clustering of speakers. The clusters obtained define time intervals with the aim to find acoustic observations from a sole speaker and to avoid overlapped speech. The algorithm was tested on the RT07s dataset obtaining an improvement of $10 \%$ relative DER on the single distant microphone condition and $4 \%$ relative in a situation where an enhanced signal is used for diarization.

\section{References}

[1] Reynolds, D. A., "Approaches and Applications of Audio Diarization", in Proc. ICASSP, 2005

[2] "The Rich Transcription 2007 Meeting Recognition Evaluation ", http://www.nist.gov/speech/tests/rt/2007/ workshop/RT07-NIST-Overview.pdf

[3] Kemp T., Schmidt, M., Westphal M., Waibel A., "Strategies for automatic segmentation of audio data", in Proc. ICASSP, pp. 1423-1426, 2000

[4] Ajmera, J. and Wooters C., "A robust speaker clustering algorithm", in Proc. ASRU'03, 2003.

[5] Tranter S. and Reynolds, D. A., "Speaker diarization for broadcast news", in Proc. ODISSEY'04, 2004

[6] Anguera, X., Wooters, C., Hernando, J., ”Acoustic Beamforming for Speaker Diarization of Meetings", in IEEE Trans. on Audio, Speech and Language Processing, Vol. 15 , No.7, pp. 2011-2022, 2007

[7] Koh, E. C. W. and et al., "Speaker Diarization Using Direction of Arrival Estimate and Acoustic Feature Information: The $\mathrm{I}^{2} \mathrm{R}$ NTU Submission for the NIST RT 2007 Evaluation ", in LNCS Springer-Verlag, CLEAR 2007 and RT 2007, Vol. 4625, 2008

[8] Wooters, C. and Huygbregts M., "The ICSI RT07s Speaker Diarization System “, in LNCS Springer-Verlag, CLEAR 2007 and RT 2007, Vol. 4625, 2008

[9] Anguera, X., Wooters, C. and Hernando, J., "Friends and enemies: a novel initialization for speaker diarization", in Proc. Interspeech, pp 689-692, 2006

[10] M.S. Brandstein and H.F. Silverman, "A Robust Method for Speech Signal Time-Delay Estimation in Reverberant Rooms“, in Proc. IEEE ICASSP, 1997.

[11] Segura, C., Abad, A., Hernando J. and Nadeu C., "Multispeaker Localization and Tracking in Intelligent Environments", in Proc. of the second International CLEAR Evaluation Workshop 2007, Springer LNCS, Vol. 4625, 2007

[12] M. Swartling et al., "Direction of Arrival Estimation for Multiple Speakers Using Time-Frequency Orthogonal Signal Separation", in Proc. of ICASSP, vol. IV, pp. 833-836, 2006.

[13] Bar-Shalom, Y., Fortman,T.E., "Tracking and Data association", Academic Press, 1988.

[14] Luque, J., Anguera X., Temko, A., Hernando, J., "Speaker Diarization for Conference Room: The UPC RT07s Evaluation System", LNCS Springer-Verlag, Vol. 4625, pp. 543-553, CLEAR 2007 and RT 2007, 2008 